RESEARCH ARTICLE

\title{
Mapping non-laminar proton acceleration in laser-driven target normal sheath field
}

\author{
C. Y. Qin ${ }^{1,2}$, H. Zhang ${ }^{1,3}$, S. Li $^{1}$, S. H. Zhai ${ }^{4}$, A. X. Li ${ }^{1,5}$, J. Y. Qian ${ }^{1}$, J. Y. Gui ${ }^{1}$, F. X. Wu ${ }^{1}$, Z. X. Zhang ${ }^{1}$, \\ Y. Xu ${ }^{1,3}$, X. Y. Liang ${ }^{1,3}$, Y. X. Leng ${ }^{1,3}$, B. F. Shen ${ }^{1,4}$, L. L. Ji ${ }^{1,3}$, and R. X. Li ${ }^{1,3,5}$ \\ ${ }^{1}$ State Key Laboratory of High Field Laser Physics, Shanghai Institute of Optics and Fine Mechanics, Chinese Academy of Sciences, \\ Shanghai 201800, China \\ ${ }^{2}$ Center of Materials Science and Optoelectronics Engineering, University of Chinese Academy of Sciences, Beijing 100049, China \\ ${ }^{3}$ CAS Center for Excellence in Ultra-intense Laser Science, Shanghai 201800, China \\ ${ }^{4}$ Department of Physics, Shanghai Normal University, Shanghai 200234, China \\ ${ }^{5}$ ShanghaiTech University, Shanghai 201210, China \\ (Received 27 August 2021; revised 25 October 2021; accepted 19 November 2021)
}

\begin{abstract}
We report on experimental observation of non-laminar proton acceleration modulated by a strong magnetic field in laser irradiating micrometer aluminum targets. The results illustrate the coexistence of ring-like and filamentation structures. We implement the knife edge method into the radiochromic film detector to map the accelerated beams, measuring a source size of 30-110 $\mu \mathrm{m}$ for protons of more than $5 \mathrm{MeV}$. The diagnosis reveals that the ring-like profile originates from low-energy protons far off the axis whereas the filamentation is from the near-axis high-energy protons, exhibiting nonlaminar features. Particle-in-cell simulations reproduced the experimental results, showing that the short-term magnetic turbulence via Weibel instability and the long-term quasi-static annular magnetic field by the streaming electric current account for the measured beam profile. Our work provides direct mapping of laser-driven proton sources in the spaceenergy domain and reveals the non-laminar beam evolution at featured time scales.
\end{abstract}

Keywords: knife-edge technique; laser-plasma interaction; non-laminar proton source; target normal sheath acceleration; Weibel instability

\section{Introduction}

Laser-driven target normal sheath acceleration (TNSA) is a very robust mechanism for generating proton sources at tens of megaelectronvolts energy with ultralow transverse emittance (e.g., better than $0.004 \mathrm{~mm} \cdot \mathrm{mrad}$ in Ref. [1]). Such highly directional proton beams are advantageous in various applications such as probing high-energy-density states $^{[2]}$, treating cancer therapy ${ }^{[3]}$, and fast ignition fusion ${ }^{[4]}$. As the TNSA facilitates compact, stable, and affordable proton sources ${ }^{[5]}$, full understanding of the beam properties becomes crucial. Usually, the proton beams accelerated from TNSA expand into vacuum in a self-similar manner ${ }^{[6]}$ and are of high degree of laminarity ${ }^{[1]}$. In other words,

Correspondence to: H. Zhang, B. F. Shen, and L. L. Ji, State Key Laboratory of High Field Laser Physics, Shanghai Institute of Optics and Fine Mechanics, Chinese Academy of Sciences, Shanghai 201800, China. Email: zhanghui1989@siom.ac.cn (H. Zhang); bfshen@mail.shcnc.ac.cn (B. F. Shen); jill@siom.ac.cn (L. L. Ji) protons at a same position have identical transverse velocities and their orbits do not cross each other ${ }^{[7]}$. Laminar beams have outstanding transport properties that can support micro-sized spot sizes, a highly favored feature in sequential applications.

However, proton beams can be significantly modulated via, for instance, Weibel-like instability (WI) during laserplasma interaction ${ }^{[8-10]}$. Experimental and simulation studies show that pre-plasma can be created by laser pre-pulse on the target rear surface ${ }^{[8,10,11]}$ or the front ${ }^{[12]}$. WI or electron filamentation instability spontaneously arises from laserdriven fast electrons streaming in background plasma ${ }^{[13-16]}$. The electron beam profile is further mapped onto the proton beam during acceleration ${ }^{[10,17]}$. Such filamentations could be diagnosed either from the spatially modulated proton beams accelerated by the sheath field ${ }^{[17,18]}$ or the transition radiation induced by fast electrons ${ }^{[19]}$. It is usually associated with strong magnetic field $(B \sim 100 \mathrm{MG})$ as probed via magnetooptic polarograms ${ }^{[20]}$. 


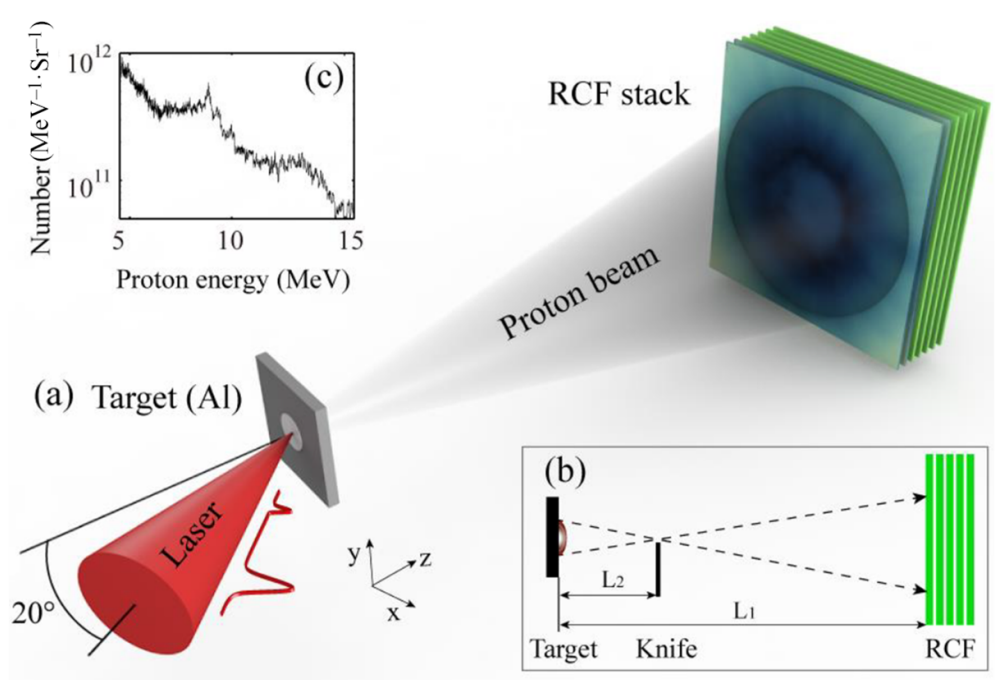

Figure 1. Sketch of the experimental setup (a) without the knife edge and (b) with the knife edge. (c) The typical proton energy spectrum of $10-\mu \mathrm{m}$ aluminum detected by a Thomson parabolic spectrometer in a separate run. The laser pulse with $1.8 \times 10^{20} \mathrm{~W} / \mathrm{cm}^{2}, 35 \mathrm{fs}, 12 \mu \mathrm{m}$ (beam size) and $10^{-6} @ 3$ ns irradiates a $10-\mu \mathrm{m}$-thick aluminum foil at $20^{\circ}$ incident angle.

A filamented profile is regarded as a key sign of disrupted laminarity ${ }^{[8,10]}$. These signals can be regularly obtained using radiochromic film (RCF) detectors. To identify whether these modulated protons are from near-axis or off-axis and further reveal the size of the perturbed area, we propose to implement the knife-edge technique (KET) ${ }^{[21]}$ into the RCF detector in laser-proton acceleration. For laminar sources, the KET would give a virtual source size in the sense of a beam waist ${ }^{[22]}$. However, it perfectly serves our purpose because we focus on non-laminar proton feature existing in acceleration.

\section{Experimental results}

Using the KET-RCF method, we experimentally study the proton distribution from laser irradiating micro-sized planar aluminum targets. We observe a ring-like profile and filamentation simultaneously. The KET diagnosis reveals that the ring structure stems from low-energy protons located far off-axis, whereas the filamentation belongs to the highenergy group disturbed by WI in the near-axis area. Typical source sizes of the non-laminar protons are found to be 30-110 $\mu \mathrm{m}$ for energy of more than $5 \mathrm{MeV}$. We believe through particle-in-cell (PIC) simulations that the magnetic field evolving at two distinctive time scales is responsible for the observed features.

\subsection{Experimental setup}

The experiments were carried out in the Shanghai Superintense Ultrafast Laser Facility (SULF). Therein, the 1-PW laser system ${ }^{[23]}$ delivers a 28 -J and 35 -fs (full width at half maximum, FWHM) pulse of wavelength $\lambda=800 \mathrm{~nm}$. The pulse is focused to a beam size of $12 \mu \mathrm{m}$ (FWHM, contains $\sim 7.2 \mathrm{~J}$ energy), yielding a peak intensity of $1.8 \times 10^{20} \mathrm{~W} / \mathrm{cm}^{2}$ on target. The laser amplified spontaneous emission (ASE) pedestal is at $10^{-11}-10^{-10}$ level and there exists a $10^{-6}$ prepulse at $3 \mathrm{~ns}$ prior to the main pulse. This strong nanosecond pre-pulse tends to trigger a strong shock which will lead to pre-plasma at both sides of the target ${ }^{[24]}$. The experimental setup is shown in Figure 1, where the laser pulse is focused onto a $10-\mu \mathrm{m}$-thick aluminum target by an $F / 3$ off-axis parabola (OAP). It is p-polarized at an incident angle of $20^{\circ}$. The RCF stack is enwrapped in $10-\mu \mathrm{m}$-thick aluminum foil and fixed behind the target at $L_{1}=94 \mathrm{~mm}$ along the target normal direction. Two types of RCF, HD-V2 and EBT3, are used in the stack with HD-V2 placed in the front ${ }^{[25]}$. A metal $(\mathrm{Cu} / \mathrm{Al}) \mathrm{knife}$ edge with $200 \mu \mathrm{m}$ thickness and $3.8 \mathrm{~mm}$ width is located at $L_{2}=12.0 \pm 1.5 \mathrm{~mm}$ behind the target. Its vertical location can be adjusted depending on whether the KET is employed.

\subsection{Ring and filamentation profiles}

We first remove the knife edge and let the protons fly freely towards the RCF stack. The signals at different proton energies are collected in Figure 2 (see full RCF signals in the Supplemental Material). Two featured structures appear in the proton spatial distribution. A clear ring profile is observed for low-energy protons $(\sim 1 \mathrm{MeV})$ in Figure 2(a1), peaking at a radius of approximately $0.8 \mathrm{~cm}$ on the RCF sheet. This corresponds to an emitting angle of about $4.8^{\circ}$, as shown in the M-shaped angular distribution in Figure 2(c). A series of experimental investigations implied that the ringlike profile could form when relativistic transparency occurs in nanometer targets ${ }^{[26-28]}$ or is deflected by the rear toroidal magnetic field formed by hot electrons in micrometer-sized targets ${ }^{[29]}$. 


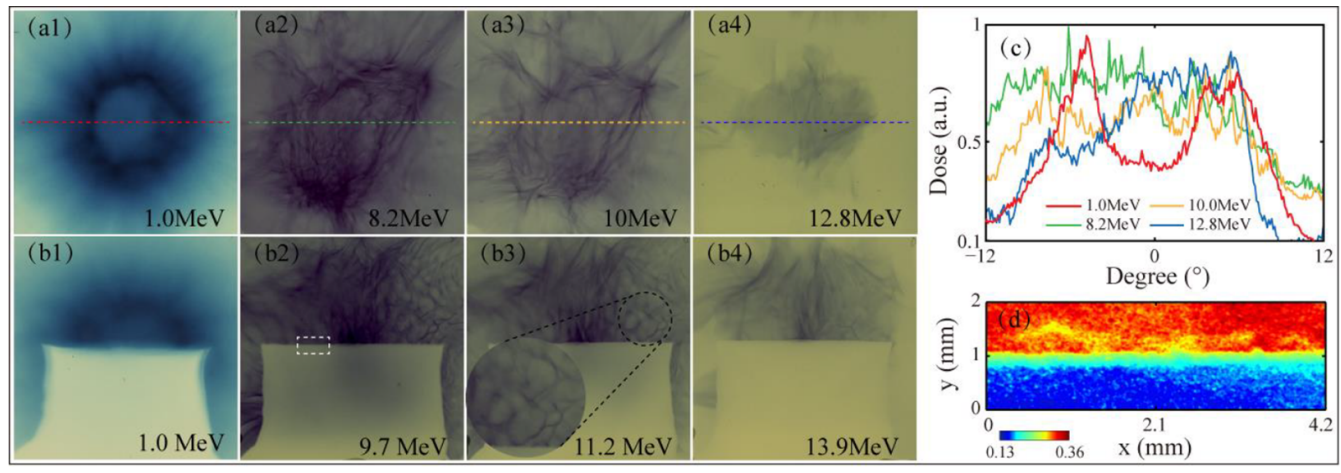

Figure 2. (a1)-(a4) The proton beam profiles of different energy on RCF without knife edge and (b1)-(b4) proton beam profiles of different energy on RCF with a knife edge. (c) The dose distribution along the dashed lines in (a1)-(a4). (d) The OD distribution in the white dashed rectangle in (b2). The red/blue parts denote the area irradiated/non-irradiated by protons and the transition between (yellow-green) is the penumbra region.

For more energetic protons $(>3 \mathrm{MeV})$, the distribution shows significant filamentation in Figures 2(a2)-2(a4). From the angular distribution in Figure 2(c), one can see that the proton beam divergence is around $20^{\circ}-24^{\circ}$ at various proton energies up to $10 \mathrm{MeV}$. This is in contrast to highdegree-laminarity proton beam flow produced in TNSA, where the beam divergence is much smaller for higher proton energies ${ }^{[1]}$. In other words, protons here are emitted along different directions with similar divergence, forming a nonlaminar beam source. The featured length scale of the filaments can be inferred from Figure 2(b3). To this end, the virtual origin of the beam source is identified to be approximately $130 \mu \mathrm{m}$ away from the target front surface using a mesh grid $^{[30]}$ (see the Supplemental Material for more information). The filament 'bubble' diameters are 1-3 mm, indicating a filament period of $\lambda_{w}=1.4-4.1 \mu \mathrm{m}$.

\subsection{Non-laminar proton beam}

We further diagnose the non-laminar proton beam employing the KET to retrieve the proton source sizes at different energies. The experimental setup is shown in Figure 1(b), where we move the knife edge up to the position that is horizontal to the target center. The RCF stack used in these shots consists of two HD-V2s and eight EBT3s. Proton signals are shown in Figures 2(b1)-2(b4) where the rectangles denote the knife shadows. The proton dose distribution can be divided into three parts. The area completely irradiated by the proton source forms the dark region, whereas the non-irradiated area corresponds to the bright region. Protons pass through the knife edge following the dashed lines in Figure 1(b) producing a penumbra area in the RCF. It is a narrow strip sandwiched between the bright and dark. We take an approximately $4 \mathrm{~mm} \times 2 \mathrm{~mm}$ knife edge area from Figure 2(b2) and show the optical density (OD) distribution in Figure 2(d). The pictures used for data processing are grayscale images scanned with a resolution of 4800 dpi. We see a clear transition area in the color map. Here the electron and X-ray irradiation background have been subtracted. The dose in the bright area is not exactly zero because the blade is not thick enough to block all protons.

Now that the width of the penumbra is known, the source size can be inferred through geometric proportional relationship. We randomly select five $x$-positions in Figure 2(d) and average the OD to remove noise. The edge spread function (ESF) of the OD value is plotted in Figure 3(a). The width of the penumbra is defined as $d=2\left(y_{1}-y_{2}\right)$, where $y_{1}$ is the relative position corresponding to the OD value of $\mathrm{od}_{1}+\left(\mathrm{od}_{2}-\mathrm{od}_{1}\right) / 4$ and $y_{2}$ to $\mathrm{od}_{2}-\left(\mathrm{od}_{2}-\mathrm{od}_{1}\right) / 4^{[21]}$. The magnification factor from source to image is simply the ratio $M \approx\left(L_{1}-L_{2}\right) / L_{2}$, about 6.8 in our setup. The source size $D$ is therefore given by $d / M$, which is $78 \pm 7 \mu \mathrm{m}$ for $9.8 \pm 0.2 \mathrm{MeV}$ protons. In general, the definition of the proton source size here is slightly larger than the FWHM value.

By implementing the KET, each RCF sheet not only records the beam profile but also the source size at a particular proton energy level. The data of the three shots with the same KET location are presented in Figure 3(b) after being averaged. Here, EBT3 sheets were mainly analyzed to avoid the influence from different RCF sensibilities. Measurements at the high-energy end give $30-50 \mu \mathrm{m}$ source size for proton energy greater than $10 \mathrm{MeV}$. The size diagnosed here $(>10 \mathrm{MeV})$ is about 3-7 times the laser spot size in FWHM. Furthermore, to confirm the KET results, we used the mesh method to calibrate the source sizes ${ }^{[30]}$. The mesh is placed parallel to the target rear surface at a distance of approximately $150 \mu \mathrm{m}$, and Figure 3(b) shows the sizes are about 43 and $25 \mu \mathrm{m}$ for the energy of 11.1 and $12.7 \mathrm{MeV}$, respectively (see the details in the Supplemental Material), in good agreement with the KET-RCF measurement. We observe a linear dependence of the source size on the proton energy of more than $5 \mathrm{MeV}$, namely protons of higher energies have smaller source sizes. This characteristic is also consistent with the reports in Refs. [31,32] using a direct surface image (DSI) method. 

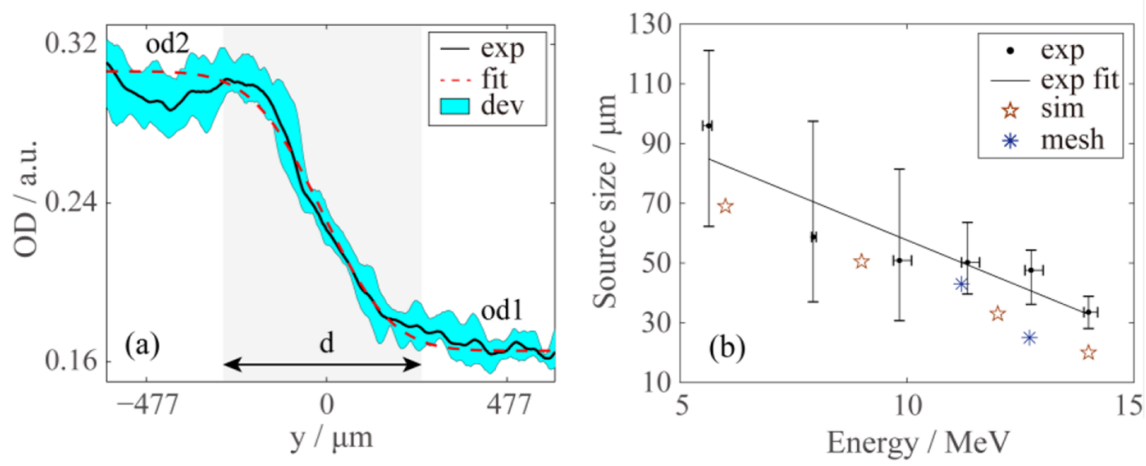

Figure 3. (a) The ESF function for the OD value along the direction of the vertical knife edge. The red dashed line shows the fitting curve of the averaged experiment results (black solid line) with $d$ the penumbra width. Data range at different $x$-positions is denoted by dev. (b) Proton source size with regards to different energies. Black line denotes linear fitting for experimental results. Blue stars represent the measurement using the mesh method and red pentagrams represent the simulation results at $t=600 \mathrm{fs}$. Horizontal error bars are determined by the RCF uncertainty and different stack combinations. The longitudinal errors result from three shoots.

\section{Simulation and discussion}

One possible mechanism responsible for the ring-like structure is the quasi-static annular magnetic field generated when laser ablates the target surface. Magnetic field up to $10^{3} \mathrm{~T}$ can be established through laser-driven electric current and protons could be diverged or focused when passing through $^{[33]}$. On the other hand, the filamentation structure is believed to be caused by WI accompanied with target-rear pre-plasma ${ }^{[8,10,11]}$. In this case, the scale length should be larger than the critical value $L_{p, c} \approx 0.13 \lambda\left(a_{0}\right)^{1 / 2} \sim 0.3 \mu \mathrm{m}^{[10]}$.

However, it is not clear why both features co-exist, with low energy protons distributed in a ring and high energy ones filamented simultaneously. Even more interesting is that the latter seems originating from the near-axis center of the interaction area thanks to the KET measurement. PIC simulations are therefore performed to reveal the underlying mechanism. We run the simulations in two dimensions using the EPOCH code $^{[34]}$. The simulation box is $100 \mu \mathrm{m} \times$ $100 \mu \mathrm{m}$ in $x \times y$ directions $(x=0-100 \mu \mathrm{m}, y=-45-$ $55 \mu \mathrm{m})$ with $8000 \times 6250$ cells. The laser pulse parameters are set to match the laboratory laser condition, which is of $0.8-\mu \mathrm{m}$ wavelength, 35 -fs duration, and $12-\mu \mathrm{m}$ spot size. It is p-polarized and incident onto the target at an angle of $20^{\circ}$. The laser intensity is $2 \times 10^{20} \mathrm{~W} / \mathrm{cm}^{2}$. The interaction target is fully ionized with initial electron temperature $10 \mathrm{eV}$. The particle-per-cell number is set to 9 . The main target is a $7-\mu \mathrm{m}$ slab (aluminum) with electron density of $80 n_{c}\left(n_{c}=\right.$ $m_{e} \varepsilon_{0} \omega^{2} / e^{2}$ is the critical density). The simulative density is set lower than the real target density in experiments. It is chosen to balance between the heavy computational load to resolve solid density plasmas and the highly overcritical condition. The target front is located at $x=10 \mu \mathrm{m}$ in the box. The electron density decreases from $50 n_{c}$ with a scale length of $1.6 \mu \mathrm{m}$ on the target front and $30 n_{c}$ with $1.0-\mu \mathrm{m}$ scale length on the rear surface, according to our hydrodynamic simulation using the FLASH code in two dimensions ${ }^{[35]}$ under the experimental pre-pulse condition (see the details in the Supplemental Material). Protons from contaminates of the aluminum target follow the same density profile, but with much lower density $\left(5.8 n_{c}\right.$ in the front and $5.3 n_{c}$ at the rear). The simulation time is defined as $0 \mathrm{fs}$ when the laser is incident from the left boundary.

When the main pulse arrives at the pre-plasma in the front, it efficiently drives energetic electrons. The latter pass through the target and stream in the pre-plasma at the rear surface. Return current grows ${ }^{[11]}$ and drives WI, leading to magnetic field filamentation ${ }^{[20,36-39]}$, as seen in the nearaxis area in Figure 4(a) at 120 fs. The transverse motion direction of the accelerated protons is disturbed when they pass through such a magnetic field. In every filament cycle $B_{z}$ changes its sign, and therefore protons are deflected in the opposite transverse direction. From Figure 4(e) we see that the transverse momenta are staggered such that their orbits intersect later in this vicinity and form a strong non-laminar flow. At 400 fs, the WI-induced magnetic field vanishes as shown in Figure 4(c). The proton density already forms significant filaments, with featured spatial period about $1.3 \mu \mathrm{m}$ (Figure 4(f)). From Figures 4(b) and 4(d) we can see that background electron shows filamentation after a longer time of evolution. As the plasma expands further, the magnetic field from filamentation decays whereas the large-scale magnetic field from the thermoelectric effect is sustained for a much longer time. Theoretical analysis suggests that the Weibel separated filament thickness should be of the order

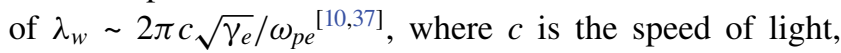
$\gamma_{e} \sim a_{0} / \sqrt{2}$ is the estimated relativistic Lorentz factor of electrons ${ }^{[40]}$, and $\omega_{p e}=\left(n_{e} e^{2} / \varepsilon_{0} m_{e}\right)^{1 / 2}$ is the local plasma frequency. In our setup, $\lambda_{w} \sim 2.1 \mu \mathrm{m}$ at critical density theoretically, which is consistent with the experimental estimate and simulations.

In our simulations, magnetic filamentation occurs at approximately $100 \mathrm{fs}$, boosting the field strength to $10^{4} \mathrm{~T}$ in less than $100 \mathrm{fs}$. The field then rapidly drops to 

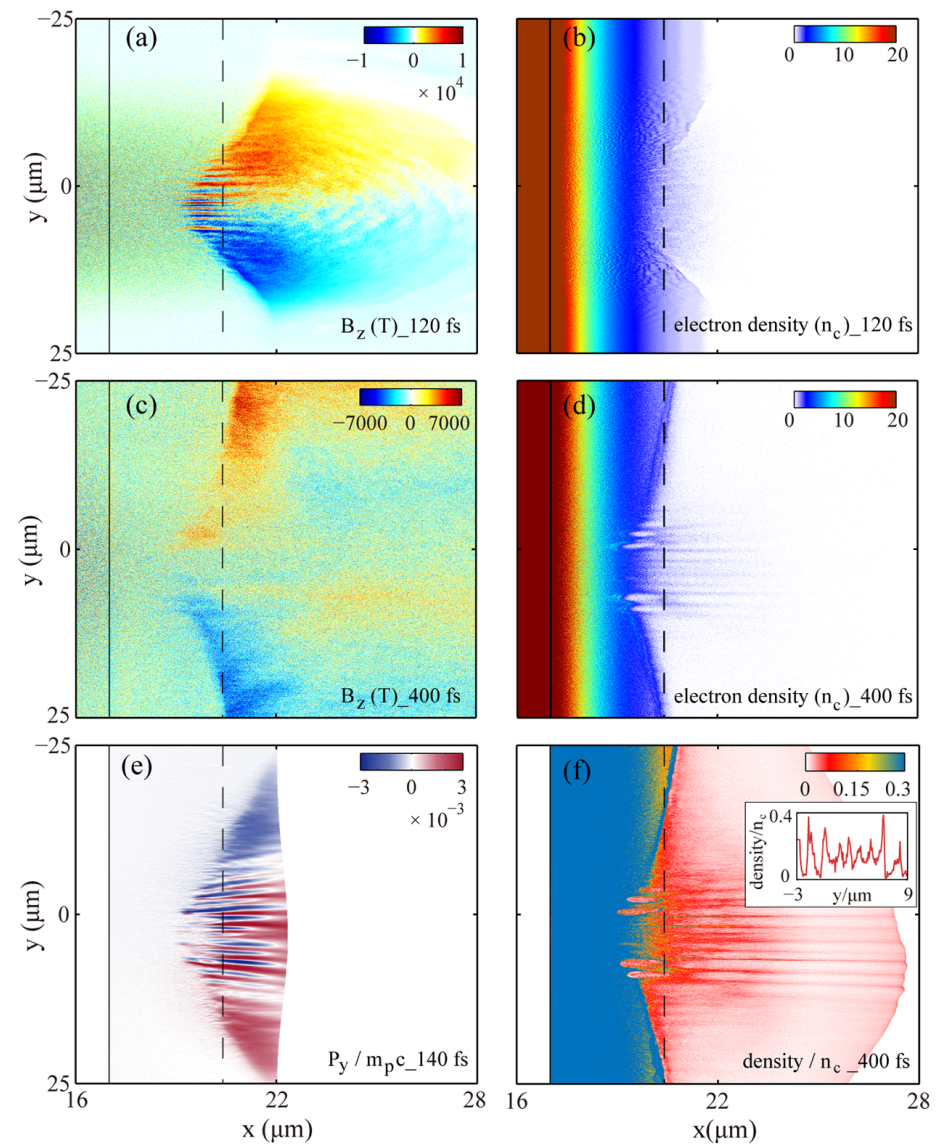

Figure 4. Results from PIC simulations. The magnetic field distribution along the $z$-direction at the rear side of the target at (a) $t=120 \mathrm{fs}$ and (c) $t=$ $400 \mathrm{fs}$. The electron density distribution at the rear side at (b) $t=120 \mathrm{fs}$ and (d) $t=400 \mathrm{fs}$. (e) The normalized transverse momentum of protons at $t=140 \mathrm{fs}$. (f) The proton density normalized to $n_{c}$ at $t=400 \mathrm{fs}$. The solid lines represent the boundary of the main target whereas the dashed lines represent the critical-density location in pre-plasma.
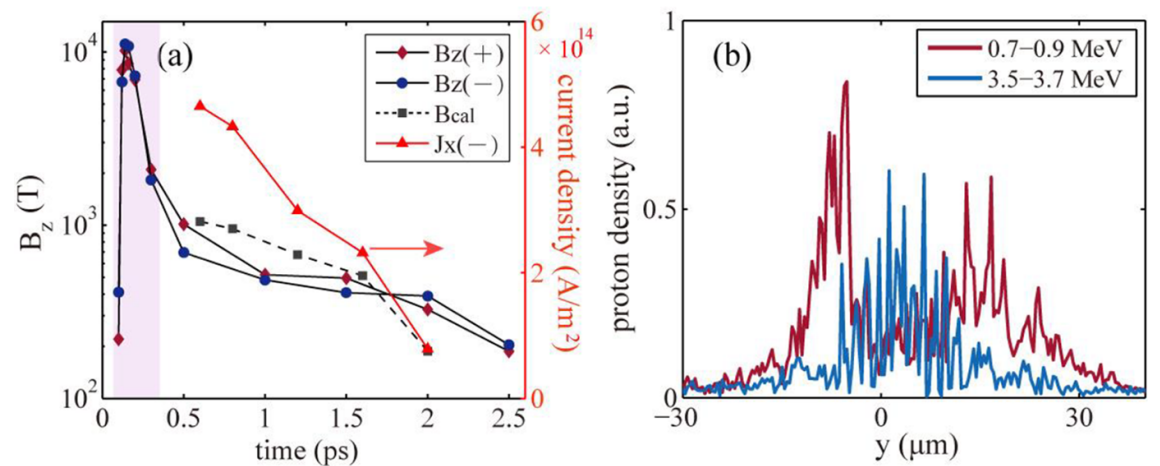

Figure 5. (a) Evolution of the $B_{z}$ field at the rear target side as a function of time. Here $B_{c a l}$ is calculated from the longitudinal net electron current density $J_{x}$ and the lilac area represents the filamentation window. (b) The transverse profile of proton density at $t=1.4 \mathrm{ps}$.

approximately $10^{3} \mathrm{~T}$, which can be seen in Figure 5(a). On the other hand, a long-term azimuthal magnetic field of hundreds of tesla persists in the far-axis area; see Figures 4(a), 4(c), and 5(a). The strong quasi-static $B$-fields are mainly generated by laser-driven electric currents, which last for picoseconds before getting damped ${ }^{[33,41]}$. Figure 5(a) shows the net current density $J_{x}$ along the $-x$ direction declining in about $1 \mathrm{ps}$. The corresponding magnetic field is estimated using $B_{c a l} \sim \mu_{0} J_{x} r_{0} / 2$, with $r_{0} \sim 4 \mu \mathrm{m}$ the radius of the electron stream. The field strength agrees with that taken directly from simulations. We note that this average current density is only about $1 \%$ of that from the laser-driven electric current or approximately $10^{16} \mathrm{~A} / \mathrm{m}^{2}$, suggesting that it is significantly neutralized by the return current. As the current decays, the $B$-field will not disappear immediately, thanks to the thermoelectric effect ${ }^{[41,42]}$. 
This magnetic field could extend to 3-5 $\mu \mathrm{m}$ length scale and provides a bending force that deflects the far-axis protons (usually of low energy) sideways. Essentially, they form a ring-like structure in space. When the $1 \mathrm{MeV}$ protons pass through a uniform magnetic field (consider $B_{z} \sim 800 \mathrm{~T}$, lasting $\sim 2 \mathrm{ps}$ ), they could be deflected to the $4.4^{\circ}$ position which is roughly consistent with the experiment peak position $4.8^{\circ}$ shown in Figure 2(c). These together lead to the proton density distribution in Figure 5(b). At 1.4 ps we find that low-energy protons $(0.7-0.9 \mathrm{MeV})$ exhibit an M-shaped profile, whereas the high-energy group (3.53.7 MeV) exhibit no collective lateral movement but filamentation. The simulation results are in good agreement with the experimental observation in Figure 2(c). We calculate the transverse dimensions of protons with different energies in the acceleration phase which is close to the real size on the target bulk. The agreement between simulations and experiments is illustrated in Figure 3(b).

\section{Conclusion}

In conclusion, through direct mapping of protons with the KET-RCF method and PIC simulations, we have found that energetic electrons streaming in the pre-plasma at the rear surface of the target induce femtosecond-lifetime filamented magnetic field and picosecond quasi-static azimuthal magnetic field. The latter is responsible for the ring-like structure of low-energy protons whereas the former is responsible for the filamentation of high-energy protons. These constitute a non-laminar proton source. The measurement developed here probably supports utilization in characterizing the ion sources employing nanometer targets which are prone to unstable disturbances ${ }^{[26,43]}$. For TNSA, our work suggests that pre-plasma on the target rear should be avoided if laminar beams are expected.

\section{Acknowledgments}

This work was supported by the Strategic Priority Research Program of the Chinese Academy of Sciences (No. XDB16), National Natural Science Foundation of China (Nos. 11875307, 11935008, 11804348, 11705260, 11905278, and 11975302), and Youth Innovation Promotion Association of the Chinese Academy of Sciences.

\section{Supplementary Materials}

To view supplementary material for this article, please visit https://dx.doi.org/10.1017/hpl.2021.54.

\section{References}

1. T. E. Cowan, J. Fuchs, H. Ruhl, A. Kemp, P. Audebert, M. Roth, R. Stephens, I. Barton, A. Blazevic, E. Brambrink, J. Cobble, J. Fernandez, J. C. Gauthier, M. Geissel, M.
Hegelich, J. Kaae, S. Karsch, G. P. L. Sage, S. Letzring, M. Manclossi, S. Meyroneinc, A. Newkirk, H. Pepin, and N. R. LeGalloudec, Phys. Rev. Lett. 92, 204801 (2004).

2. L. Romagnani, J. Fuchs, M. Borghesi, P. Antici, P. Audebert, F. Ceccherini, T. Cowan, T. Grismayer, S. Kar, A. Macchi, P. Mora, G. Pretzler, A. Schiavi, T. Toncian, and O. Willi, Phys. Rev. Lett. 95, 195001 (2005).

3. S. V. Bulanov, T. Z. Esirkepov, V. S. Khoroshkov, A. V. Kuznetsov, and F. Pegoraro, Phys. Lett. A 299, 240 (2002).

4. M. Roth, T. E. Cowan, M. H. Key, S. P. Hatchett, C. Brown, W. Fountain, J. Johnson, D. M. Pennington, R. A. Snavely, S. C. Wilks, K. Yasuike, H. Ruhl, F. Pegoraro, S. V. Bulanov, E. M. Campbell, M. D. Perry, and H. Powell, Phys. Rev. Lett. 86, 436 (2001).

5. J. G. Zhu, M. J. Wu, Q. Liao, Y. X. Geng, K. Zhu, C. C. Li, X. H. Xu, D. Y. Li, Y. R. Shou, T. Yang, P. J. Wang, D. H. Wang, J. J. Wang, C. E. Chen, X. T. He, Y. Y. Zhao, W. J. Ma, H. Y. Lu, T. Tajima, C. Lin, and X. Q. Yan, Phys. Rev. Accel. Beams 22, 061302 (2019).

6. P. Mora, Phys. Rev. Lett. 90, 185002 (2003).

7. S. Humphries, Charged Particle Beams (Wiley, New Jersey, 1990).

8. G. G. Scott, C. M. Brenner, V. Bagnoud, R. J. Clarke, B. Gonzalez-Izquierdo, J. S. Green, R. I. Heathcote, H. W. Powell, D. R. Rusby, B. Zielbauer, P. McKenna, and D. Neely, New J. Phys. 19, 043010 (2017).

9. K. Quinn, L. Romagnani, B. Ramakrishna, G. Sarri, M. E. Dieckmann, P. A. Wilson, J. Fuchs, L. Lancia, A. Pipahl, T. Toncian, O. Willi, R. J. Clarke, M. Notley, A. Macchi, and M. Borghesi, Phys. Rev. Lett. 108, 135001 (2012).

10. S. Göde, C. Rödel, K. Zeil, R. Mishra, M. Gauthier, F. E. Brack, T. Kluge, M. J. MacDonald, J. Metzkes, L. Obst, M. Rehwald, C. Ruyer, H. P. Schlenvoigt, W. Schumaker, P. Sommer, T. E. Cowan, U. Schramm, S. Glenzer, and F. Fiuza, Phys. Rev. Lett. 118, 194801 (2017).

11. M. Tatarakis, F. N. Beg, E. L. Clark, A. E. Dangor, R. D. Edwards, R. G. Evans, T. J. Goldsack, K.W. D. Ledingham, P. A. Norreys, M. A. Sinclair, M. S. Wei, M. Zepf, and K. Krushelnick, Phys. Rev. Lett. 90, 175001 (2003).

12. J. Metzkes, T. Kluge, K. Zeil, M. Bussmann, S. D. Kraft, T. E. Cowan, and U. Schramm, New J. Phys. 16, 023008 (2014).

13. R. Lee and M. Lampe, Phys. Rev. Lett. 31, 1390 (1973).

14. P. McKenna, A. P. L. Robinson, D. Neely, M. P. Desjarlais, D. C. Carroll, M. N. Quinn, X. H. Yuan, C. M. Brenner, M. Burza, M. Coury, P. Gallegos, R. J. Gray, K. L. Lancaster, Y. T. Li, X. X. Lin, O. Tresca, and C. G. Wahlstrom, Phys. Rev. Lett. 106, 185004 (2011).

15. D. A. MacLellan, D. C. Carroll, R. J. Gray, N. Booth, M. Burza, M. P. Desjarlais, F. Du, B. Gonzalez-Izquierdo, D. Neely, H. W. Powell, A. P. L. Robinson, D. R. Rusby, G. G. Scott, X. H. Yuan, C. G. Wahlstrom, and P. McKenna, Phys. Rev. Lett. 111, 095001 (2013).

16. M. S. Wei, F. N. Beg, E. L. Clark, A. E. Dangor, R. G. Evans, A. Gopal, K. W. D. Ledingham, P. McKenna, P. A. Norreys, M. Tatarakis, M. Zepf, and K. Krushelnick, Phys. Rev. E 70, 056412 (2004).

17. J. Fuchs, T. E. Cowan, P. Audebert, H. Ruhl, L. Gremillet, A. Kemp, M. Allen, A. Blazevic, J.-C. Gauthier, M. Geissel, M. Hegelich, S. Karsch, P. Parks, M. Roth, Y. Sentoku, R. Stephens, and E. M. Campbell, Phys. Rev. Lett. 91, 255002 (2003).

18. R. J. Dance, N. M. H. Butler, R. J. Gray, D. A. MacLellan, D. R. Rusby, G. G. Scott, B. Zielbauer, V. Bagnoud, H. Xu, A. P. L. Robinson, M. P. Desjarlais, D. Neely, and P. McKenna, Plasma Phys. Control. Fusion 58, 014027 (2016).

19. M. Storm, A. A. Solodov, J. F. Myatt, D. D. Meyerhofer, C. Stoeckl, C. Mileham, R. Betti, P. M. Nilson, T. C. Sangster, 
W. Theobald, and C. Guo, Phys. Rev. Lett. 102, 235004 (2009).

20. S. Mondal, V. Narayanan, W. J. Ding, A. D. Lad, B. Hao, S. Ahmad, W. M. Wang, Z. M. Sheng, S. Sengupt, P. Kaw, A. Das, and G. R. Kumar, Proc. Natl. Acad. Sci. U.S.A 109, 8011 (2012).

21. C. Ekdahl, J. Opt. Soc. Am. A 28, 2501 (2011).

22. M. Roth, M. Allen, P. Audebert, A. Blazevic, E. Brambrink, T. E. Cowan, J. Fuchs, J.-C. Gauthier, M. Geibell, M. Hegelich, S. Karsch, J. Meyer-ter-Vehn, H. Ruhl, T. Schlegel, and R. B. Stephens, Plasma Phys. Control. Fusion 44, B99 (2002).

23. Z. X. Zhang, F. X. Wu, J. B. Hu, X. J. Yang, J. Y. Gui, P. H. Ji, X. Y. Liu, C. Wang, Y. Q. Liu, X. M. Lu, Y. Xu, Y. X. Leng, R. X. Li, and Z. Z. Xu, High Power Laser Sci. Eng. 8, e4 (2020).

24. O. Lundh, F. Lindau, A. Persson, C. G. Wahlstrom, P. McKenna, and D. Batani, Phys. Rev. E 76, 026404 (2007).

25. http://www.gafchromic.com/gafchromic-film/radiotherapyfilms/index.asp.

26. B. Gonzalez-Izquierdo, M. King, R. J. Gray, R. Wilson, R. J. Dance, H. Powell, D. A. Maclellan, J. McCreadie, N. M. H. Butler, S. Hawkes, James S. Green, C. D. Murphy, L. C. Stockhausen, D. C. Carroll, N. Booth, G. G. Scott, M. Borghesi, D. Neely, and P. McKenna, Nat. Commun. 7, 12891 (2016).

27. D. Jung, B. J. Albrightl, L. Yin, D. C. Gautier, R. Shah, S. Palaniyappan, S. Letzring, B. Dromey, H. C. Wu, T. Shimada, R. P. Johnson, M. Roth, J. C. Fernandez, D. Habs, and B. M. Hegelich, New J. Phys. 15, 123035 (2013).

28. H. W. Powell, M. King, R. J. Gray, D. A. MacLellan, B. Gonzalez-Izquierdo, L. C. Stockhausen, G. Hicks, N. P. Dover, D. R. Rusby, D. C. Carroll, H. Padda, R. Torres, S. Kar, R. J. Clarke, I. O. Musgrave, Z. Najmudin, M. Borghesi, D. Neely, and P. McKenna, New J. Phys. 17, 103033 (2015).

29. Y. Murakami, Y. Kitagawa, Y. Sentoku, M. Mori, R. Kodama, K. A. Tanaka, K. Mima, and T. Yamanaka, Phys. Plasmas 8, 9 (2001).

30. M. Borghesi, A. J. Mackinnon, D. H. Campbell, D. G. Hicks, S. Kar, P. K. Patel, D. Price, L. Romagani, A. Schiavi, and O. Willi, Phys. Rev. Lett. 92, 055003 (2004).

31. M. Roth, P. Audebert, A. Blazevic, E. Brambrink, J. Cobble, T. E. Cowan, J. Fernandez, J. Fuchs, M. Geissel, M. Hegelich, S. Karsch, H. Ruhl, M. Schollmeier, and R. Stephens, Opt. Commun. 264, 519 (2006).

32. E. Brambrink, J. Schreiber, T. Schlegel, P. Audebert, J. Cobble, J. Fuchs, M. Hegelich, and M. Roth, Phys. Rev. Lett. 96, 154801 (2006).
33. G. Sarri, A. Macchi, C. A. Cecchetti, S. Kar, T. V. Liseykina, X. H. Yang, M. E. Dieckmann, J. Fuchs, M. Galimberti, L. A. Gizzi, R. Jung, I. Kourakis, J. Osterholz, F. Pegoraro, A. P. L. Robinson, L. Romagnani, O. Willi, and M. Borghesi, Phys. Rev. Lett. 109, 205002 (2012).

34. T. D. Arber, K. Bennett, C. S. Brady, A. Lawrence-Douglas, M. G. Ramsay, N. J. Sircombe, P. Gillies, R. G. Evans, H. Schmitz, A. R. Bell, and C. P. Ridgers, Plasma Phys. Control. Fusion 57, 113001 (2015).

35. B. Fryxell, K. Olson, P. Ricker, F. X. Timmes, M. Zingale, D. Q. Lamb, P. MacNeice, R. Rosner, J. W. Truran, and H. Tufo, The Astrophys. J. Suppl. Ser. 131, 273 (2000).

36. W. Fox, G. Fiksel, A. Bhattacharjee, P. Y. Chang, K. Germaschewski, S. X. Hu, and P. M. Nilson, Phys. Rev. Lett. 111 225002 (2013).

37. C. Ruyer, S. Bolaños, L. Lancia, M. Nakatsutsumi, B. Albertazzi, S. N. Chen, L. Romagnani, R. Shepherd, P. Antici, J. Böker, V. Dervieux, M. Swantusch, M. Borghesi, O. Willi, H. Pépin, M. Starodubtsev, M. Grech, C. Riconda, L. Gremillet, and J. Fuchs, Nat. Phys. 16, 983 (2020).

38. C. M. Huntington, F. Fiuza, J. S. Ross, A. B. Zylstra, R. P. Drake, D. H. Froula, G. Gregori, N. L. Kugland, C. C. Kuranz, M. C. Levy, C. K. Li, J. Meinecke, T. Morita, R. Petrasso, C. Plechaty, B. A. Remington, D. D. Ryutov, Y. Sakawa, A. Spitkovsky, H. Takabe, and H. S. Park, Nat. Phys. 11, 173 (2015).

39. M. King, N. M. H. Butler, R. Wilson, R. Capdessus, R. J. Gray, H. W. Powell, R. J. Dance, H. Padda, B. GonzalezIzquierdo, D. R. Rusby, N. P. Dover, G. S. Hicks, O. C. Ettlinger, C. Scullion, D. C. Carroll, Z. Najmudin, M. Borghesi, D. Neely, and P. McKenna, High Power Laser Sci. Eng. 7, e14 (2019).

40. S. C. Wilks, A.B. Langdon, T. E. Cowan, M. Roth, M. Singh, S. Hatchett, M. H. Key, D. Pennington, A. Mackinnon, and R. A. Snavely, Phys. Plasmas 8, 542 (2001).

41. B. Albertazzi, E. d'Humières, L. Lancia, V. Dervieux, P. Antici, J. Böcker, J. Bonlie, J. Breil, B. Cauble, S. N. Chen, J. L. Feugeas, M. Nakatsutsumi, P. Nicolaï, L. Romagnani, R. Shepherd, Y. Sentoku, M. Swantusch, V. T. Tikhonchuk, M. Borghesi, O. Willi, H. Pépin, and J. Fuchs, Rev. Sci. Instrum. 86, 043502 (2015).

42. R. J. Mason and M. Tabak, Phys. Rev. Lett. 80, 524 (1998).

43. C. A. J. Palmer, J. Schreiber, S. R. Nagel, N. P. Dover, C. Bellei, F. N. Beg, S. Bott, R. J. Clarke, A. E. Dangor, S. M. Hassan, P. Hilz, D. Jung, S. Kneip, S. P. D. Mangles, K. L. Lancaster, A. Rehman, A. P. L. Robinson, C. Spindloe, J. Szerypo, M. Tatarakis, M. Yeung, M. Zepf, and Z. Najmudin, Phys. Rev. Lett. 108, 225002 (2012). 